\section{ORIGINAL RESEARCH}

M. Li

H.G. He

W. Shi

J. Li

B. LV

C.H. Wang

Q.W. Miao

Z.C. Wang

N.L. Wang

M. Walter

B.A. Sabel

\title{
Quantification of the Human Lateral Geniculate Nucleus In Vivo Using MR Imaging Based on Morphometry: Volume Loss with Age
}

\begin{abstract}
BACKGROUND AND PURPOSE: Because it is a small subcortical structure, the precise measurement of the human LGN is still a technical challenge. In this article, we identify the LGN in vivo, measure its volume based on high-resolution MR imaging, and then relate its volume to subject age to evaluate the potential clinical application
\end{abstract}

MATERIALS AND METHODS: A semiautomatic LGN isolation method was developed on scans obtained with 1.5T MR imaging, which involves highlighting the surrounding landmarks, obtaining candidate LGN voxels with a region-growing algorithm, and isolating the LGN from the ventral diencephalon. The method was accessed with a test-retest reliability on the results from 55 healthy subjects at different ages.

RESULTS: This method showed high test-retest within-subject reliability (ICC, 0.950 and 0.948 in left and right hemispheres, respectively) among 3 independent measurements in each subject. The unilateral volume was highly variable, ranging from 52 to $102 \mathrm{~mm}^{3}$ in the left and 66 to $105 \mathrm{~mm}^{3}$ in the right hemisphere, with significantly larger volumes on the right $\left(86 \mathrm{~mm}^{3}\right)$ than on the left $\left(77 \mathrm{~mm}^{3}\right)$. The combined bilateral volumes (controlled for ICV) significantly decreased in size with progressing age from 20 to 65 years $(r=-0.512, P=.000)$. There was no sex difference in bilateral LGN volumes (male/female: $163.1 \pm 18.2 / 162.2 \pm 21.4 \mathrm{~mm}^{3}$ ).

CONCLUSIONS: Using our new technique, we were able to reliably determine the human LGN volume in vivo, which was found to decline with age. The volumes obtained by our method corresponded well with previously reported postmortem values, so our method may be considered to be superior for investigating the pathology of LGN.

ABBREVIATIONS: $\mathrm{BOLD}=$ blood oxygenation level dependent; $\mathrm{CH}-\mathrm{PC}=$ chiasmato-commissural; $I C C=$ intraclass correlation coefficient; ICV = intracranial brain volume; LGN = lateral geniculate nucleus; $\mathrm{VDC}=$ ventral diencephalon

B eing a major visual relay station for information that travels from the retina to primary visual cortex, the LGN is the first stage at which cortical top-down feedback signals affect visual processing. ${ }^{1-3}$ Although there is increasing interest in the morphometry of the $\mathrm{LGN},{ }^{4-8}$ the current imaging methods of LGN determination are not anatomically specific and the results are also inconsistent. A reliable and practicable method to precisely measure LGN in vivo is still not available.

In contrast to prior morphometric and histologic postmortem studies, ${ }^{9-11}$ volume determinations need to be easily applied in studying clinical problems such as aging or other degenerative disorders. Additionally, LGN imaging also needs to

\section{Received June 23, 2011; accepted after revision August 19}

From the State Key Laboratory of Management and Control for Complex Systems (M.L., B.L., Q.W.M., C.H.W., H.G.H.), Institute of Automation, Chinese Academy of Sciences, Beijing, China; Departments of Ophthalmology (W.S., N.L.W.) and Radiology (J.L., Z.C.W.), Beijing Tongren Hospital, Capital Medical University, China; and Departments of Psychiatry (M.W.) and Medical Psychology (B.A.S.), Otto-von-Guericke University of Magdeburg, Magdeburg, Germany.

This work was supported by the National Natural Science Foundation of China (20670530, 60875079, 81070762), Beijing Nova Plan (2007A094). M. Li was supported by Boehringer Ingelheim Fonds in Germany.

Please address correspondence to Huiguang He, PhD, State Key Laboratory of Management and Control for Complex Systems, Institute of Automation, Chinese Academy of Sciences, Beijing, 100190, China; e-mail: huiguang.he@ia.ac.cn

Indicates open access to non-subscribers at www.ajnr.org

be a highly sensitive and reliable visualization; enabling direct stereotactic target determination would substantially improve measurement accuracy.

Several technical advances in neuroimaging have emerged and have shed light on the study of subcortical structures in vivo. Horton et $\mathrm{al}^{12}$ provided the first images of the LGN by proton density-weighted imaging on postmortem examinations and in living human subjects. They reported a good correlation of MR images with anatomic sections and concluded that it is possible to unequivocally identify the LGN by using MR imaging; they also provided a suggested range for the human LGN volume. Recently, Gupta et $\mathrm{al}^{8}$ studied patients with glaucoma and reported that the LGN height was significantly decreased; this decrease was also shown by coronal proton density MR imaging. However, there has been a special concern for the lack of sufficient contrast to differentiate thalamic nuclei, which have various relaxation times but few proton attenuations. ${ }^{13}$

fMRI can also be used to indirectly image the LGN, though activation measurements only reflect the collective responses of neural populations in the LGN, which are associated with visual stimuli. For example, Fujita et $\mathrm{al}^{14}$ reported that the fMRI activation region could be superimposed onto the LGN that was anatomically identified by proton density-weighted images. O'Connor et $\mathrm{al}^{4}$ reported that the locations of the activations were consistent with the anatomic locations of the LGN as determined on high-resolution anatomic MR images. 
However, LGN functional imaging has been constrained by technical limitations, such as the fact that the activation level is close to noise and that the activation area in the LGN probably depends on the global normalization procedure ${ }^{15}$ and also on the visual activity status. So the activation of BOLD signal intensity is especially problematic in clinical populations in which changes in the cerebrovascular system can be influenced by age or impaired vision. ${ }^{16-18}$ Moreover, there is still controversy over the approximated volumes of the LGN and whether the activated volume readings are, in part, influenced by activation from adjacent structures such as the pulvinar during visual stimulation. ${ }^{19,20}$

A recent study ${ }^{21}$ introduced a feasible procedure to identify the human LGN primarily by well-known landmarks with 3D T1-weighted images. On the basis of this understanding, we have now developed a semiautomatic method to isolate the LGN on T1-weighted MR images with image enhancement and cortex parcellation mapping. The validity and reliability of this method were documented in 55 healthy controls at different ages. As we now show, LGN volumes obtained with this method agree reasonably with the reported anatomic postmortem volume. In addition, the results also showed for the first time significant LGN volume shrinkage in normal human aging, which suggests that age-related vision loss has a central component.

\section{Materials and Methods}

\section{Patients and MR Imaging Acquisition}

The 55 healthy controls (29 men, 26 women; ages, 20-67 years, mean, $38.6 \pm 14.4$ years) were recruited and studied at the Beijing Tongren Hospital, Capital Medical University. All subjects underwent eye examinations and visual field testing by using standard perimetric tests to exclude the possibility of visual system dysfunction. Subjects with a history of ocular or neurologic disease were also excluded. The study was approved by the ethics committee of the Beijing Tongren Hospital. Written informed consent was obtained from each subject according to the provisions of the Declaration of Helsinki.

All measurements were performed on a 1.5T Signa MR imaging scanner (GE Healthcare, Milwaukee, Wisconsin). Each subject was first scanned by using a T2-weighted fluid-attenuated inversion recovery sequence $(\mathrm{TR}=9000 \mathrm{~ms}, \mathrm{TE}=120 \mathrm{~ms}, \mathrm{TI}=2125 \mathrm{~ms}, \mathrm{FOV}=$ $240 \times 210 \mathrm{~mm}$, matrix size $=256 \times 222$, thirty-two sections, $4.0-\mathrm{mm}$ section thickness with $0.8-\mathrm{mm}$ intersection gap) to detect any brain abnormalities. A set of high-resolution axial T1-weighted images was acquired with a 3D fast-spoiled gradient sequence by using the following scan parameters: $\mathrm{TR}=10 \mathrm{~ms}, \mathrm{TE}=4.4 \mathrm{~ms}, \mathrm{TI}=600 \mathrm{~ms}$, $\mathrm{FOV}=260 \times 260 \mathrm{~mm}$, matrix size $=256 \times 256$, number of excitations $=1$, yielding 152 sections and a voxel dimension of $1 \times 1 \times 1 \mathrm{~mm}$.

Image enhancement and cortex parcellation techniques were used to isolate the LGN on 3D T1-weighted images. First, nonuniformity correction and gray-level normalization was used to enhance the raw image for visualization. Then anatomic landmarks were highlighted by adjusting image intensity and color mapping. Second, a regiongrowing algorithm was used to obtain the candidate voxels of LGN. Then a VDC mask was applied to exclude the non-LGN voxels, and subsequently the LGN voxels were obtained as the intersection of candidate voxels and the VDC (Fig 1).

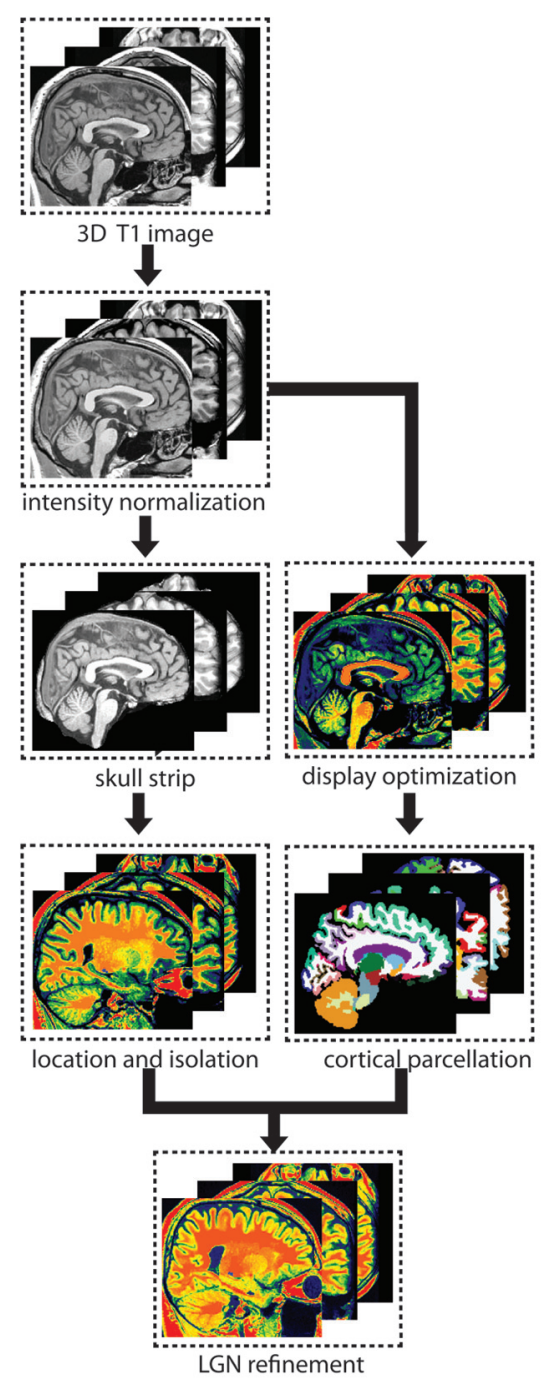

Fig 1. Pipeline of LGN isolation on a 3D T1-weighted image. The procedure mainly included intensity nonuniformity correction, gray-level normalization, color-mapping highlighting, cerebral cortex parcellation, landmark identification, and LGN localization and refinement.

\section{Image Preprocessing}

Intensity normalization was performed to remove variations due to magnetic susceptibility artifacts and radio-frequency field inhomogeneities on individual MR imaging volumes by using the N3 algorithm. ${ }^{22}$ The intensity of in-plane voxels was also normalized and resampled into 8-bit gray levels.

\section{LGN Localization and Measurement}

The boundary of the LGN was defined in accordance with an adapted procedure of Korsholm et $\mathrm{al}^{21}$ and entailed the following steps:

1) Setting of the display threshold and color-map scheme

In the aforementioned T1 image (Fig $2 A-C$ ), the minimum display threshold was set at 40, and a built-in Adobe color table scheme was chosen within MRIcron software (http://www.cabiatl.com/ mricro). As shown in Fig $2 D-F$, the examined nucleus had a relatively homogeneous structure, and it can be isolated from surrounding matter.

2) Identification of assisting anatomic landmarks

After placing the cursor point only in the posterior commissureobex plane on parasagittal images (Fig 2D), the LGN in both hemispheres was located, with the help of the posterior commissure and 

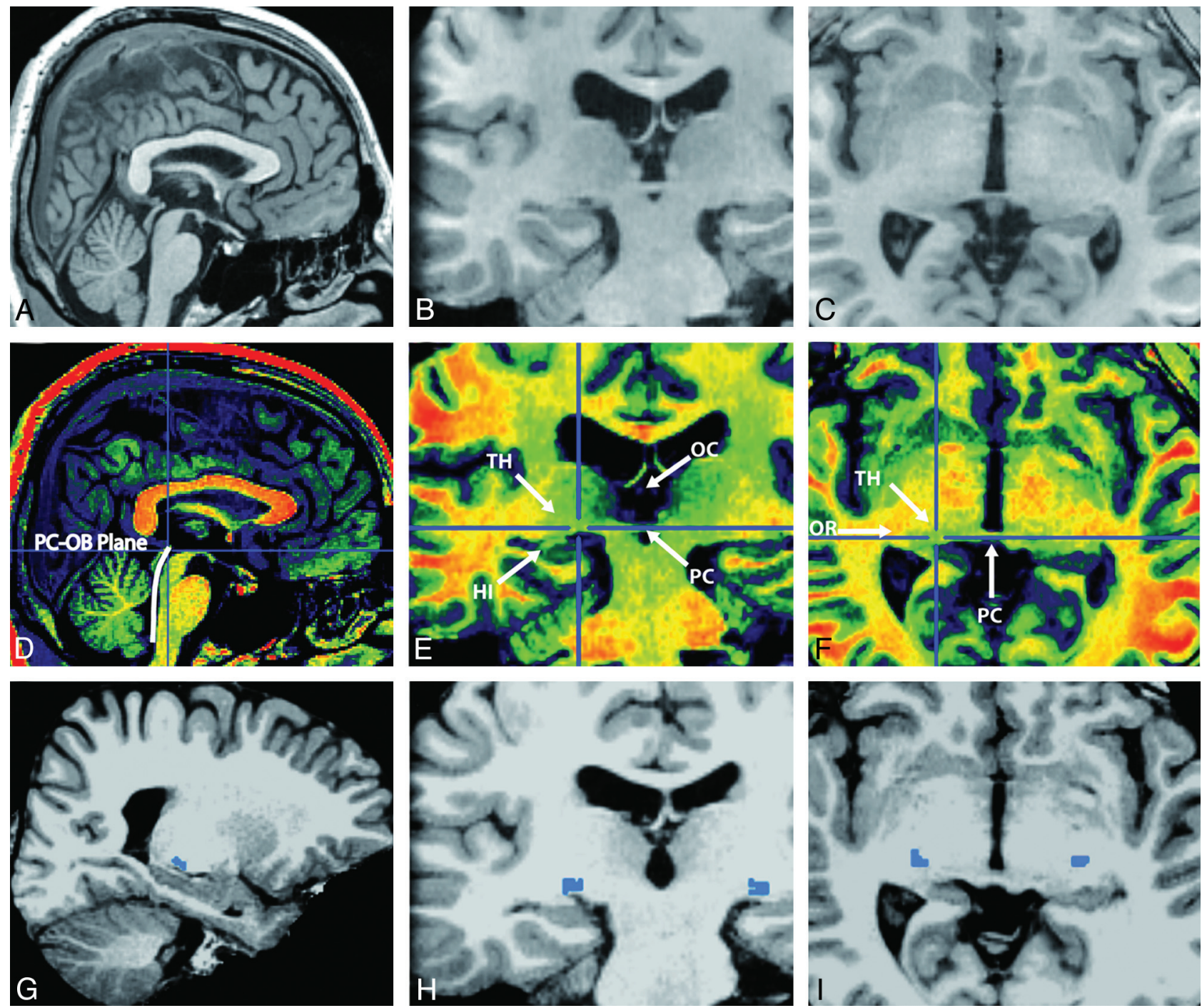

Fig 2. Delineation of the LGN on cross-sectional images. $A-F$, The images show the cerebral cortex in gray levels $(A-C)$ and pseudocolor enhancement images $(D-F)$. $G-I$, The cross-sectional images of candidate LGN maps are shown in blue.

hippocampus, on coronal images (Fig 2E), and the CH-PC and optic tract, on the axial view (Fig $2 F)$, as suggested by previous studies. ${ }^{21,23,24}$ It is quite remarkable that prior enhancement was necessary to achieve the best possible homogeneity to properly examine the nucleus.

3) Delineation of LGN voxels

The boundary of the LGN was delineated on a section that was characterized by having the largest extension of the LGN. A regiongrowing algorithm was performed to obtain a continuous cluster of voxels starting from a user-defined seed point, which was positioned at the center of the LGN by the operators.

$R_{V O I}=\cup_{i=1}^{m} P_{i},(i=1,2, \ldots m, m<n)$,

$$
\text { if } I\left(P_{i}\right)-I(S)<I_{0},\left|X\left(P_{i}\right)-X(S)\right| \leq R_{0} .
$$

Here, $I$ represents the intensity of the voxel and the coordinate of the voxel as $X$. The values of the radius $R_{o}$ and the difference from the origin $I_{0}$ were determined on the basis of the experiments reported in the literature $8,12,25,26$ and our pilot trials (see "Histology study" section in Table 1 to review the radius of LGN, and Fig $2 \mathrm{H}$, -I for the obtained candidate voxel of the LGN).

4) LGN refinement with a VDC mask
Finally, the VDC (Fig 3A,-B) was used to refine the LGN voxels, since the majority of the posterior tissue in VDC is anatomically defined as the LGN. ${ }^{27}$ The VDC mask was generated from automatic subcortical segmentation and the cortical parcellation in FreeSurfer (http://surfer.nmr.mgh.harvard.edu/) and was used to refine the LGN by intersecting with the candidate voxels of the LGN $\left(R_{L G N}=R_{V O I} \cap R_{V D C}\right.$, Fig $\left.3 C,-D\right)$. To check and review the boundary of the LGN, we found the $\mathrm{CH}-\mathrm{PC}$ to be the preferable plane for visualization of the LGN in the axial view. ${ }^{23}$

An operator delineated the boundary of the LGN following the above procedure. In this study, the rating was repeated by 2 experienced neuroradiologists and a neurologist who had no knowledge of the subject's identity and other experimental information. The ratings were performed at an interval of 2 weeks, so each study subject's data were the average of 3 independent measurements by independent observers.

\section{Statistical Analysis}

All statistical analyses were conducted by using the Statistical Package for the Social Sciences, Version 16.0 (SPSS, Chicago, Illinois). An 
Table 1: Overview of LGN volumes from published studies

\begin{tabular}{|c|c|c|c|c|}
\hline Modality & Authors & No. & Cases & $\begin{array}{l}\text { Morphometric Measurements } \\
\text { (volume or height) }\end{array}$ \\
\hline \multirow[t]{4}{*}{ Histology study } & Putnam, $1926^{9}$ & 3 & Healthy subjects & Ranging from 91.9 to $157 \mathrm{~mm}^{3}$ \\
\hline & Zvorykin, $1980^{11}$ & 17 & Healthy subjects & Ranging from 66 to $152 \mathrm{~mm}^{3}$ \\
\hline & Selemon and Begovic, $2007^{5}$ & 30 & Healthy subjects & Average volume: $79.67 \mathrm{~mm}^{3}$ \\
\hline & Dorph-Petersen et al, $2005^{6}$ & 30 & Healthy subjects (only left LGN) & Average volume: $104 \mathrm{~mm}^{3}$ \\
\hline \multirow[t]{4}{*}{ fMRI study } & Chen et al, $1999^{31}$ & 8 & Healthy subjects & Activation area: $4.6 \times 4.6 \mathrm{~mm}^{2}$ \\
\hline & Kastner et al, $2004^{20}$ & 6 & Healthy subjects & Left/right: $234 \pm 29 \mathrm{~mm}^{3} / 244 \pm 28 \mathrm{~mm}^{3}$ \\
\hline & Korsholm et al, $2007^{21}$ & 19 & Patients with isolated optic neuritis & Average of bilateral LGN: $267 \pm 27 \mathrm{~mm}^{3}$ \\
\hline & Hess et al, $2009^{7}$ & 6 & Patients with amblyopia & Left/right: $409 \pm 306 \mathrm{~mm}^{3} / 439 \pm 310 \mathrm{~mm}^{3}$ \\
\hline $\begin{array}{l}\text { Proton density } \\
\text { MRI study }\end{array}$ & Gupta et al, $2009^{8}$ & 18 & 8 Healthy subjects & Height: left/right $4.83 \pm 0.95 \mathrm{~mm} / 4.74 \pm 0.54 \mathrm{~mm}$ \\
\hline
\end{tabular}

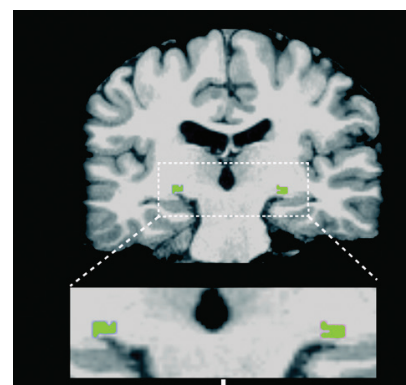

A

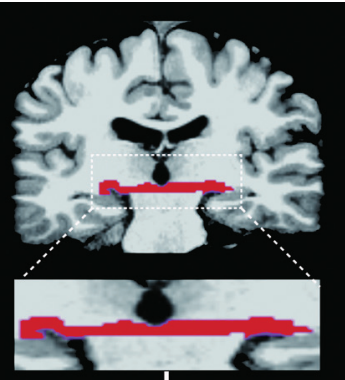

B

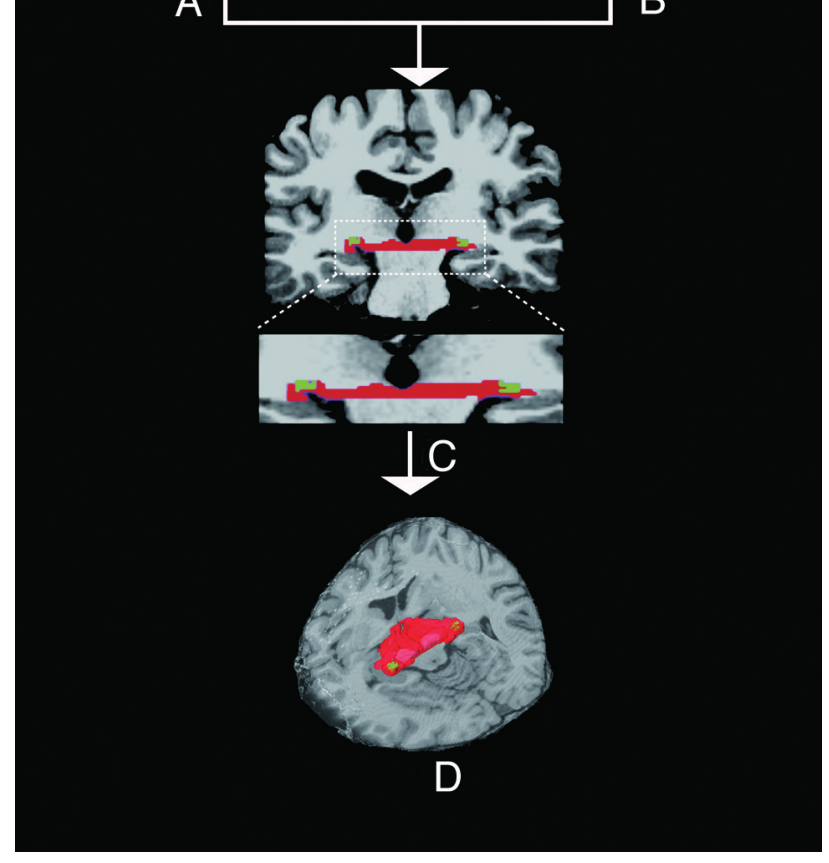

Fig 3. Definition of candidate LGN. The left and right VDC was automatically identified by the FreeSurfer on original T1 images. $A-D$, The LGN is isolated as the intersection area between the outlined VOI (candidate LGN, $A$, in green) and the VDC mask (in red); it is, $R_{L G N}=R_{V O I} \cap R_{V D C}(D, 3 D$ visualization).

analysis of the ICC was used to access the test-retest within-subject reliability. A repeated-measures ANOVA was used to compare 3 measurements in each hemisphere. Then the average volume of the 3 measurements was calculated for each LGN.

Paired comparison was performed to access the asymmetry of bilateral LGN volumes in controls. Pearson correlations were used to examine the relationship between bilateral LGN volume and age after adjusting the ICV. In an exploratory manner, the sex difference was also analyzed in our sample. All results were expressed as mean $\pm \mathrm{SD}$. For all tests, a $P$ value $<.05$ was considered significant.

\section{Results}

Expert validation confirmed semiautomated LGN detection in all cases. None of the detected $2 \times 55$ structures had to be excluded according to image inspection as described above.

Test-retest reliability of repeated morphometric measurements of the LGN with/without the VDC mask was investigated in all subjects. Compared with the results without the VDC mask, the results obtained with the VDC mask revealed high test-retest within-subject reliability (without the VDC mask: left LGN, ICC $=0.824$; right $\mathrm{LGN}$, ICC $=0.796$; with VDC mask: left LGN, ICC $=0.950$; right $\mathrm{LGN}, \mathrm{ICC}=0.948$ ).

The repeated-measures ANOVA was used to calculate possible differences of volume estimates bilaterally across 3 measurements (left LGN, $F=1.906, P=.154$; right LGN, $F=$ $0.431, P=.489$; Greenhouse-Geisser corrected; Table 2).

Mean LGN volume of $83 \mathrm{~mm}^{3}$ (left hemisphere, 52-102 $\mathrm{mm}^{3}$; mean, $77 \mathrm{~mm}^{3}$; right hemisphere, $66-105 \mathrm{~mm}^{3}$; mean, $86 \mathrm{~mm}^{3}$ ) was consistent with that in the postmortem studies of Putnam, ${ }^{9}$ who reported volumes from 77 to $115 \mathrm{~mm}^{3}$ and Zvorykin, ${ }^{10,11}$ who reported volumes from 66 to $152 \mathrm{~mm}^{3}$.

Combined bilateral LGN volumes were found to decrease with age $(r=-0.512, P=.000$, Fig 4$)$ after regressing out brain sizes as ICV. We found no sex difference in LGN volumes by a 2 -sided $t$ test (male, $163.114 \pm 18.230 \mathrm{~mm}^{3}$; female, $\left.162.2179 \pm 21.439 \mathrm{~mm}^{3} ; t=0.168, P=.867\right)$.

On an exploratory level, LGN sizes were compared bilaterally and left-right correspondence was explored. The average LGN volume of the group was significantly larger on the right than on the left side (left, $76.478 \pm 14.310 \mathrm{~mm}^{3}$; right, $86.212 \pm 11.096 \mathrm{~mm}^{3}$, difference $=9.733 ; t=4.389, P=$ .000). However, no correlations were found between the LGN volumes in both hemispheres $(r=0.181, P=.186)$.

\section{Discussion}

We have developed, for the first time, a semiautomatic method to isolate the LGN, which is capable of estimating the volume on the basis of high-resolution MR structural images. The robustness of this method was validated by 55 healthy controls ranging in age from 20 to 67 years. The main findings were that LGN volumes range from 52 to $102 \mathrm{~mm}^{3}$ (mean, 77 $\mathrm{mm}^{3}$ ) in the right and $66-105 \mathrm{~mm}^{3}$ (mean, $86 \mathrm{~mm}^{3}$ ) in the left hemisphere. These values agree reasonably well with the reported volumes in postmortem studies. ${ }^{9,11}$ Thus, MR structural imaging is a valid and reliable method to evaluate LGN volume in vivo, when used in combination with our semiau- 


\begin{tabular}{|c|c|c|c|c|c|c|c|c|c|}
\hline \multirow[b]{2}{*}{ Condition } & \multirow[b]{2}{*}{ Hemi } & \multicolumn{3}{|c|}{ Repeated Measurement (mean) } & \multicolumn{2}{|c|}{$\begin{array}{c}\text { Mauchly } \\
\text { Sphericity } \\
\text { Test }\end{array}$} & \multicolumn{3}{|c|}{$\begin{array}{c}\text { Greenhouse-Geisser } \\
\text { Correction }\end{array}$} \\
\hline & & $1 \mathrm{st}$ & 2nd & $3 r d$ & W & Sig. & $\epsilon$ & $F$ & Sig. \\
\hline \multirow[t]{2}{*}{ LGN volume before mask filtering } & Left & $103.455 \pm 10.561$ & $104.473 \pm 11.825$ & $104.182 \pm 12.412$ & - & - & - & - & - \\
\hline & Right & $105.709 \pm 11.512$ & $105.018 \pm 11.176$ & $104.527 \pm 13.179$ & - & - & - & - & - \\
\hline \multirow[t]{2}{*}{ LGN volume after mask filtering } & Left & $77.200 \pm 14.213$ & $76.945 \pm 14.431$ & $75.290 \pm 16.311$ & 0.691 & 0.000 & 0.764 & 1.906 & 0.154 \\
\hline & Right & $86.255 \pm 10.729$ & $85.691 \pm 11.068$ & $86.691 \pm 13.043$ & 0.430 & 0.000 & 0.637 & 0.431 & 0.489 \\
\hline
\end{tabular}

Note:- Hemi indicates hemisphere; W, Mauchly's W value; Sig., significance; $\epsilon$, estimating sphericity; -, no statistical analysis.

a As it was shown in this table, the obtained LGN from the region-growing algorithm may contain pixels that belong to neighboring tissues, such as the thalamus, hippocampus, or CSF. The repeated-measures ANOVA analysis was performed on the results filtered by the VDC mask in the following part of this article.

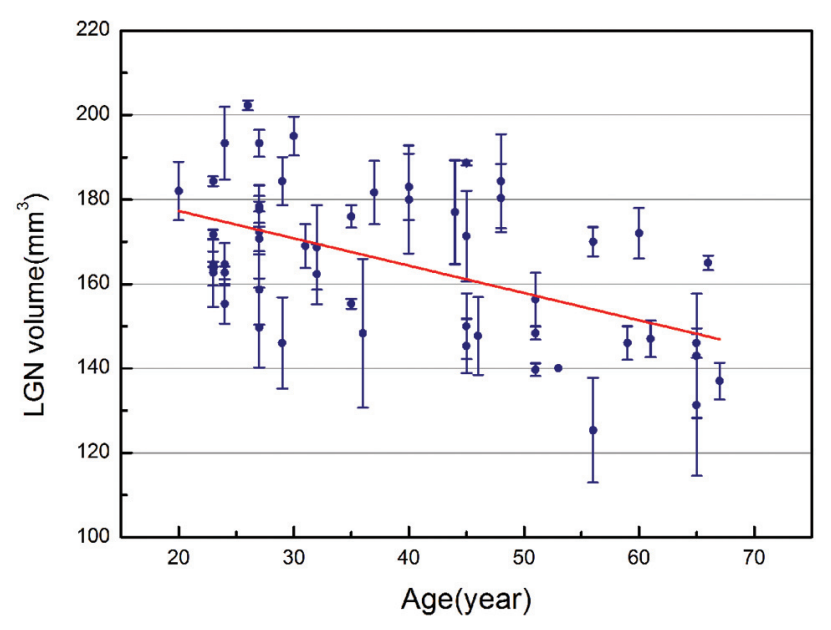

Fig 4. Relationship between bilateral LGN volume and age in all subjects $(n=55)$. Bilateral LGN volume significantly decreases with the age of the subjects (correlation of $r=$ $-0.512, P=.000$; volume-controlled for intracranial volume).

tomatic analysis method. In addition, we demonstrated LGN volume shrinkage in normal aging (see below).

The LGN is a major relay station of the retinofugal pathway, modulating the transmission of visual information that travels to the visual cortex. The characterization of the normal LGN and possible degenerative changes after nervous system damage are still a matter of debate, from a clinical as well as a methodologic point of view (Table 1). However, the precise determination of LGN in vivo is technically challenging due to its relatively small size and subcortical location.

In our study, LGN volumes were found to be comparable with volumes obtained by other postmortem studies. For example: Zvorykin ${ }^{11}$ reported a 2 - to 3 -fold volume range (66$152 \mathrm{~mm}^{3}$ ) among 17 individuals. Putnam 9 reported volumes of $77-115 \mathrm{~mm}^{3}$ but only studied 3 individuals. Andrews et $\mathrm{al}^{28}$ studied 15 specimens and determined the volume in the right (91.1-154 $\mathrm{mm}^{3}$; mean, $121 \mathrm{~mm}^{3}$ ) and left hemispheres (91.9$157 \mathrm{~mm}^{3}$; mean, $\left.115 \mathrm{~mm}^{3}\right)$. They also reported a 2- to 3-fold variation in the size of LGN between subjects.

Rather than using postmortem tissue, we developed a more reliable method to measure the LGN, which enables direct stereotactic target determination. Measurements done in vivo would substantially improve accuracy and aid in observing pathologic changes. The results obtained in our study are consistent with the postmortem results, ${ }^{9,11}$ showing comparable estimates of the average LGN volume and a high degree of variability (approximately 2 -fold) with good test-retest reliability and consistency (Table 2).
Other studies have used proton density-weighted images ${ }^{12}$ and fMRI to determine LGN volume in vivo. ${ }^{8,16,28}$ The proton density images were found to be susceptible to changes in water content and generally provided good visualization of white matter but poor visualization of gray matter. However, differences in relaxation times and too low proton density among thalamic nuclei caused low contrast and quantitative uncertainties. ${ }^{13}$ The activation of neuronal populations in the LGN associated with visual stimuli is an alternative method to estimate LGN volume in fMRI studies, but fMRI experiments are much more involved, hence less practical, for routine clinical application.

There is considerable controversy about the precise relationship of the presumed LGN activated area and the actual volumes (Table 1). Due to the partial volume effects and the activation from adjacent structures such as the pulvinar, ${ }^{20}$ BOLD-based activation of the LGN inevitably introduces bias to measurements. In addition, the activation scope of the LGN is close to noise level and most likely depends on the global normalization procedure. ${ }^{15}$ In extreme clinical cases, where permanent visual impairment or even blindness occurs, with fMRI methods, it is very difficult to acquire reliable data. Such issues have harmed the consensus of fMRI results and ultimately more widespread application in visual system research with human subjects.

Manual delineation of the LGN might produce more accurate results. However, this would be extremely time-consuming, and it is potentially prone to subjective bias, especially when the native image itself is of low-quality resolution or contrast. Our semiautomatic method improves the precision and speed of a prior region-of-interest-based method of Korsholm et $\mathrm{al}^{21}$ by using the FreeSurfer subcortical pipeline. ${ }^{29,30}$ This pipeline is fully automated and user-independent because it uses a neighborhood function to encode spatial information, a forward model of the MR scanner parameters to improve sequence independence, and a nonlinear function to account for morphologic differences between the atlas and the individual brain. With the help of the obtained VDC mask in FreeSurfer, it is possible to easily exclude non-LGN voxels, which originate mainly from the hippocampus, white matter, or CSF.

Kastner et $\mathrm{al}^{20}$ approximated the LGN as a cube with a mean side length at $4.89 \pm 0.27 \mathrm{~mm}$, which is consistent with the results by Chen et al. ${ }^{31}$ In addition, this closely matches the observations of Gupta et $\mathrm{al},{ }^{8}$ who estimated the side length at $4.74 \pm 0.54$ and $4.83 \pm 0.95 \mathrm{~mm}$ for the right and left LGN, respectively. Therefore, a sphere VOI with a $3-\mathrm{mm}$ radius was 
used as a mask to extract the LGN after the region-growing algorithm in this study. Moreover, we also noted that the posterior commissure usually appeared in the same section together with the LGN. By following the optic tract, it was also possible to trace of the LGN in the coronal plane.

With this new LGN imaging method, our study investigated the effects of aging on LGN volumes to test the value of our procedure in a medical context. Because each LGN represents only one-half of the visual fields, we summed up and compared the bilateral LGN volumes, accounting for visual input from each eye. The bilateral LGN volumes in our samples decreased with age $(r=-0.512, P=.000)$ after regressing out ICV. This is an important observation because it might indicate that the loss of vision with progressing age may also have a brain-based component. However, further study of age-related changes is needed to demonstrate that our method is sufficiently sensitive to monitor longitudinal changes such as degeneration in the LGN. In fact, this semiautomatic method may lay the basis for further studies of the diagnosis and treatment of neurologic and ophthalmologic diseases and may also very well be useful for neurosurgical intervention.

With regard to the clinical observation of LGN volume changes with age, our general finding is consistent with those of animal studies, which have already reported LGN degeneration in normal aging. ${ }^{32-34}$ Diaz et $\mathrm{al}^{32}$ compared the volume change of the dorsal LGN as a function of age and found significant decreases of neuronal volume attenuation and numeric attenuation of neurons in old rats. Similarly, de la Roza et $\mathrm{al}^{33}$ and Vidal et $\mathrm{al}^{34}$ reported a significant reduction in the diameters of both the soma and nuclei of neurons of the dorsal LGN in aged rats. As suggested in their study, aging and partial loss of visual input appeared to induce small changes in the morphology of most of the dorsal LGN neurons. However, to the best of our knowledge, LGN volume changes associated with aging have never been studied in the human brain, and it now needs to be determined whether LGN volume loss is strictly a result of reduced afferent input from the retina or whether it is caused by LGN cell loss.

No sex difference in LGN volumes in the samples was observed, but the volume of the right LGN was, on average, larger than that of the left. Nevertheless, the difference is smaller than the variance introduced by repeated measurements. In addition, given that most subjects have 1 dominant eye, it would be of interest to determine if the asymmetry of the LGN volume is related to unilateral dominance. Additional studies are required to evaluate the relationship of LGN volume and eye dominance, including larger samples and a correlation with functional measures of vision.

In the field of neuroimaging, it is still a technical challenge to precisely visualize and distinguish the irregular and variable laminar patterns of the human LGN because this would require higher resolution. Therefore, it is imperative to apply our method to data obtained from high-resolution (7T) MR imaging, in which anatomic landmarks are more distinct and can be more easily identified.

\section{Limitations of the Current Study}

Careful attention is required when using our semiautomatic technique. We used the VDC mask from FreeSurfer to refine the non-LGN voxels in the candidate voxels from a region- growing algorithm. In the definition of VDC in FreeSurfer, the choroidal fissure is the inferior border of the LGN, and the most posterior extent of the VDC is the LGN (see more details in http://www.cma.mgh.harvard.edu/manuals/segmentation/). The subcortical segmentation in FreeSurfer takes account of the MR imaging scanner parameters, curvature and spatial information from the pial surface, and morphologic differences between the atlas and each individual. If the individual information is incorporated into the segmentation, the resulting mask and further LGN isolation would be more specific. Therefore, our results rely on the subcortical segmentation in FreeSurfer. In addition, the volume estimates are less precise and are subject to potential bias when the referred structural image itself is not well-defined.

\section{Conclusions}

Our study introduces an innovative semiautomatic method to isolate the LGN on anatomic MR images. Compared with previous studies, the measured LGN volumes agree reasonably well with the reported anatomic volume in postmortem examinations. Our method shows the possibility of assessing structural MR imaging morphologic and volume changes of the LGN in vivo. As a result, this study will greatly enhance future studies of different neurologic and ophthalmologic diseases that affect the central visual pathway, and one such example is our documentation of LGN volume shrinkage, which is associated with normal aging.

\section{Acknowledgments}

We thank Jianhong Li, MD, for assistance in reviewing our results, and Dr William Uber, Shan Yang, and Tara Crute, for helping with preparation of the manuscript.

Disclosures: Qianwen Miao-RELATED: Support for Travel to Meetings for the Study or Other Purposes: National High-Tech Research and Development Plan of China (863), grant No. 2007AA01Z327. M. Walter-RELATED: Grant. DFG-SFB779, ${ }^{*}$ Comments: government grant; UNRELATED: Grants/Grants Pending: ERA-Net "Supp-Hab".* * Money paid to institution.

\section{References}

1. McCormick DA, Feeser HR. Functional implications of burst firing and single spike activity in lateral geniculate relay neurons. Neuroscience 1990;39:103-13

2. Hendry SH, Calkins DJ. Neuronal chemistry and functional organization in the primate visual system. Trends Neurosci 1998;21:344-49

3. Dai Y, Sun X, Chen Q. Differential induction of c-Fos and c-Jun in the lateral geniculate nucleus of rats following unilateral optic nerve injury with contralateral retinal blockade. Exp Brain Res 2009;193:9-18

4. O'Connor DH, Fukui MM, Pinsk MA, et al. Attention modulates responses in the human lateral geniculate nucleus. Nat Neurosci 2002;5:1203-09

5. Selemon LD, Begovic A. Stereologic analysis of the lateral geniculate nucleus of the thalamus in normal and schizophrenic subjects. Psychiatry Res 2007;151:1-10

6. Dorph-Petersen KA, Pierri JN, Perel JM, et al. The influence of chronic exposure to antipsychotic medications on brain size before and after tissue fixation: a comparison of haloperidol and olanzapine in macaque monkeys. Neuropsychopharmacology 2005;30:1649-61

7. Hess RF, Thompson B, Gole G, et al. Deficient responses from the lateral geniculate nucleus in humans with amblyopia. Eur J Neurosci 2009;29:1064-70

8. Gupta N, Greenberg G, de Tilly LN, et al. Atrophy of the lateral geniculate nucleus in human glaucoma detected by magnetic resonance imaging. $\mathrm{Br} \mathrm{J}$ Ophthalmol 2009;93:56-60.

9. Putnam TJ. Studies on the central visual system. IV. The details of the organization of the geniculostriate system in man. Arch Neurol 1926;16:683-707

10. Zvorykin VP. Neuro morphological evidence of individual differences in human vision [in Russian]. Arkh Anat Gistol Embriol 1981;81:21-24

11. Zvorykin VP. New data on individual quantitative features of the human lateral geniculate body [in Russian]. Arkh Anat Gistol Embriol 1980;78:24-27 
12. Horton J, Landau K, Maeder P, et al. Magnetic resonance imaging of the human lateral geniculate body. Arch Neurol 1990;47:1201-06

13. Kanowski M, Voges J, Tempelmann C. Delineation of the nucleus centre median by proton density weighted magnetic resonance imaging at $3 \mathrm{~T}$. Neurosurgery 2010;66:E121-23

14. Fujita N, Tanaka $\mathrm{H}$, Takanashi $\mathrm{M}$, et al. Lateral geniculate nucleus: anatomic and functional identification by use of MR imaging. AJNR Am J Neuroradiol 2001;22:1719-26

15. Miki A, Liu C-shang J, Liu GT. Effects of voxel size on detection of lateral geniculate nucleus activation in functional magnetic resonance imaging. Jpn J Ophthalmol 2004;48:558-64

16. D'Esposito M, Deouell LY, Gazzaley A. Alterations in the BOLD fMRI signal with ageing and disease: a challenge for neuroimaging. Nat Rev Neurosci 2003;4:863-72

17. Behzadi Y, Liu TT. An arteriolar compliance model of the cerebral blood flow response to neural stimulus. Neuroimage 2005;25:1100-11

18. Lu K, Perthen JE, Duncan RO, et al. Noninvasive measurement of the cerebral blood flow response in human lateral geniculate nucleus with arterial spin labeling fMRI. Hum Brain Mapp 2008;29:1207-14

19. Chen W, Kato T, Zhu XH, et al. Mapping of lateral geniculate nucleus activation during visual stimulation in human brain using fMRI. Magn Reson Med 1998;39:89-96

20. Kastner S, Connor DH, Fukui MM, et al. Functional imaging of the human lateral geniculate nucleus and pulvinar. J Neurophysiol 2004;91:438-48

21. Korsholm K, Madsen KH, Frederiksen JL, et al. Recovery from optic neuritis: an ROI-based analysis of LGN and visual cortical areas. Brain 2007;1244-53

22. Sled JG, Zijdenbos AP, Evans AC. A nonparametric method for automatic correction of intensity nonuniformity in MRI data. IEEE Trans Med Imaging 1998;17:87-97

23. Tamraz J, Saban R, Reperant J, et al. A new cephalic reference plane for use with magnetic resonance imaging: the chiasmato-commissural plane. Surg Radiol Anat 1991;13:197-201

24. Xian JF, Zhao B, Tong YJ, et al. Study of normal human optic tract with MRI [in Chinese]. Chinese Journal of CT And MRI 2004;2:19-24

25. Andrews TJ, Halpern SD, Purves D. Correlated size variations in human visual cortex, lateral geniculate nucleus, and optic tract. J Neurosci 1997;17:2859-68

26. Barnes GG, Li X, Thompson B, et al. Decreased gray matter concentration in the lateral geniculate nuclei in human amblyopes. Invest Ophthalmol Vis Sci 2010;51:1432-38

27. Desikan RS, Se F, Fischl B, et al. An automated labeling system for subdividing the human cerebral cortex on MRI scans into gyral based regions of interest. Neuroimage 2006;31:968-80

28. Andrews TJ, Halpern SD, Purves D. Correlated size variations in human visual cortex, lateral geniculate nucleus, and optic tract. J Neurosci 1997;17:2859

29. Fischl B, Salat DH, Busa E, et al. Whole brain segmentation: automated labeling of neuroanatomical structures in the human brain. Neuron 2002;33: 341-55

30. Fischl B, Kouwe AV, Halgren E, et al. Automatically parcellating the human cerebral cortex. Cereb Cortex 2004;14:11-22

31. Chen W, Zhu XH, Thulborn KR, et al. Retinotopic mapping of lateral geniculate nucleus in humans using functional magnetic resonance imaging. Proc Natl Acad Sci U S A 1999;96:2430-34

32. Diaz F, Villena A, Gonzalez P, et al. Stereological age-related changes in neurons of the rat dorsal lateral geniculate nucleus. Anat Rec 1999;255:396-400

33. de la Roza C, Cano J, Satorre J, et al. A morphologic analysis of neurons and neuropil in the dorsal lateral geniculate nucleus of aged rats. Mech Ageing Dev 1986;34:233-48

34. Vidal L, Ru C, Villena A et al. Quantitative age-related changes in dorsal latera geniculate nucleus relay neurons of the rat. Neuroscience 2004;48:387-96 